\title{
Area law microstate entropy from criticality and spherical symmetry
}

\author{
Gia Dvali \\ Arnold Sommerfeld Center, Ludwig-Maximilians-Universität, Theresienstraße 37, 80333 München, Germany; \\ Max-Planck-Institut für Physik, Föhringer Ring 6, 80805 München, Germany; \\ and Center for Cosmology and Particle Physics, Department of Physics, New York University, \\ 726 Broadway, New York, New York 10003, USA
}

(Received 6 March 2018; published 8 May 2018)

\begin{abstract}
It is often assumed that the area law of microstate entropy and the holography are intrinsic properties exclusively of the gravitational systems, such as black holes. We construct a nongravitational model that exhibits an entropy that scales as area of a sphere of one dimension less. It is represented by a nonrelativistic bosonic field living on a $d$-dimensional sphere of radius $R$ and experiencing an angular-momentumdependent attractive interaction. We show that the system possesses a quantum critical point with the emergent gapless modes. Their number is equal to the area of a $d-1$-dimensional sphere of the same radius $R$. These gapless modes create an exponentially large number of degenerate microstates with the corresponding microstate entropy given by the area of the same $d$-1-dimensional sphere. Thanks to a double-scaling limit, the counting of the entropy and of the number of the gapless modes is made exact. The phenomenon takes place for arbitrary number of dimensions and can be viewed as a version of holography.
\end{abstract}

DOI: 10.1103/PhysRevD.97.105005

\section{INTRODUCTION}

Since the celebrated paper by Bekenstein [1], one of the main open problems in gravity is to understand the origin of the area law for the black hole entropy. This law is often viewed as a defining property of black holes, something that is linked with the most fundamental aspect of quantum gravity.

The area scaling of the black hole entropy gave rise to several hypothesis, the most well known of which goes under the name of holography [2]. Another famous conjecture revealing the holographic nature of gravity, is AdS/ CFT correspondence [3-5]. Once again, it appears that information of a gravitational system can be summed up in the degrees freedom of a lower dimensional theory $[5,6]$.

In the light of the above developments it is important to understand whether the area law entropy and holography are fundamentally linked to gravity, or represent a more general phenomenon of nature, which could take place also in nongravitational systems. This point of view was advocated in [7].

For shedding some light on the above question, it would be extremely useful to have a simple quantum modeleven if at a toy level-in which the area law scaling of

Published by the American Physical Society under the terms of the Creative Commons Attribution 4.0 International license. Further distribution of this work must maintain attribution to the author(s) and the published article's title, journal citation, and DOI. Funded by SCOAP . entropy could be unambiguously demonstrated, without being blurred by the uncontrollable quantum gravity corrections. We are not aware of any such model in the literature and it is the purpose of the present paper to construct one.

Before continuing, we would like to mention an important work [8], which gives a microscopic counting of entropy for the extremal black holes in string theory, using the power of supersymmetry. The attitude that we shall take is much less profound and does not rely on any of these properties. Of course, we shall only present a toy model, but the fact that it gives both the area-type law and holography-without any obvious involvement of gravity-is somewhat remarkable.

Therefore, in this paper we shall attempt to construct an example of a simple nongravitational quantum system which exhibits the emergence of the gapless modes with their number scaling as the area of a lower dimensional sphere.

Some clarifications of terminology are in order:

(1) First, under the term entropy we do not mean the entanglement entropy, which usually scales as the area. Rather, we mean the true micro-state entropy, i.e., the $\log$ from the number of the degenerate microstates.

(2) Second, we use the term area law despite the fact that the manifold that our field lives on is a $d$-sphere and has no space boundary. However, this term indicates that the entropy scales not as the volume of a $d$-sphere, but as the volume of $(d-1)$-sphere of the same radius, or equivalently, as the area of a boundary of the $d$-dimensional hemisphere. 
(3) Third, the term nongravitational is used solely due to the external appearance of the model, which does not seem to contain any massless spin-2 field. It may very well be (and we think this is indeed the case) that the model secretly captures some key properties of the black hole gravity, along the ideas of [7].

The model that we shall construct, describes a quantum mechanical bosonic field, $\hat{\psi}$, living in a space of a $d$ dimensional sphere of radius $R$. We show that this system exhibits a quantum critical point where many gapless modes emerge. Their number is given by the area of a $d-1$ dimensional sphere of the same radius $R$. Correspondingly, the entropy of the resulting micro-states scales as the area of the $d$-1-dimensional sphere as well.

As we shall see, the area scaling of the micro-state entropy is the result of the interplay of the two effects. These are: The quantum criticality of attractive bosons that creates large number of gapless modes, and the leveldegeneracy due to spherical symmetry that ensures that the number of gapless modes scales as area. Without both of these effects in place, the system would result neither in the area law nor in holography.

The presented model provides a supportive evidence for the hypothesis of [7], according to which the origin of the black hole entropy and holography lies in quantum criticality of attractive bosons (gravitons). It shares some similarities with the model discussed earlier in [9], but exhibits some key differences. We shall first give a detailed description of the model and later give the interpretation of our results.

\section{MODEL}

The Hamiltonian of the model has the following form:

$$
\hat{H}=-\int d^{d} \Omega \hat{\psi}^{\dagger} \Delta \hat{\psi}+g \Omega\left(\hat{\psi}^{\dagger} \Delta \hat{\psi}^{\dagger}\right)(\hat{\psi} \Delta \hat{\psi}) .
$$

Here, $d^{d} \Omega$ is a volume element of a $d$-dimensional unit sphere, with the angular coordinates $\vartheta_{a}(a=1, \ldots d)$ and the total volume $\Omega$. The operator $\Delta \equiv \frac{\hbar^{2}}{2 m R^{2}} \Delta_{d}$ is a rescaled Laplace operator, with $\Delta_{d}$ being the usual covariant Laplace operator on a $d$-dimensional unit sphere. $R$ is the radius of the proper sphere. The parameter $m$ is the mass of the boson and $g$ is a positive coupling constant. Note, $\Delta$ has the dimensionality of energy and correspondingly $g$ has the dimensionality of inverse energy.

The operator $\hat{\psi}\left(\vartheta_{a}\right)$ is a bosonic field that describes a distribution of the particle number on the $d$-sphere. We can represent it as an infinite sum over the creation and annihilation operators for the modes with different generalized angular momenta,

$$
\hat{\psi}=\sum_{k} Y_{k}\left(\vartheta_{a}\right) \hat{a}_{k}
$$

where $Y_{k}\left(\vartheta_{a}\right)$ are the spherical harmonic functions on $S_{d}$. The label $k$ stands for a set of $d$ integers $k \equiv\left(k_{1}, \ldots, k_{d}\right)$, which satisfy $\left|k_{1}\right| \leq k_{2} \leq \ldots \leq k_{d}=0,1, \ldots, \infty$. That is, $Y_{k} \equiv Y_{k_{1}, \ldots, k_{d}}$. These spherical harmonics form a complete orthonormal set. Thus, among other properties, they satisfy:

$$
\int d \Omega Y_{k}^{*} Y_{k^{\prime}}=\delta_{k k^{\prime}}
$$

where, $\delta_{k k^{\prime}} \equiv \delta_{k_{1} k_{1}^{\prime}} \delta_{k_{2} k_{2}^{\prime}} \ldots \delta_{k_{d} k_{d}^{\prime}}$. They represent the eigenfunctions of the rescaled Laplace operator

$$
\begin{aligned}
\Delta Y_{k} & =-\epsilon_{k} Y_{k}, \\
\text { where, } \quad \epsilon_{k} & \equiv \frac{\hbar^{2}}{2 m R^{2}} k_{d}\left(k_{d}+d-1\right) .
\end{aligned}
$$

The eigenvalues $\epsilon_{k}$ have the dimensionalities of energy, measured in units of the quantity $\frac{\hbar^{2}}{2 m R^{2}}$. It is very important for what follows that the level $\epsilon_{k}$ exhibits the following degeneracy,

$$
\mathcal{N}_{k}=\sum_{k_{d-1}=0}^{k_{d}} \sum_{k_{d-2}=0}^{k_{d-1}} \ldots \sum_{k_{1}=-k_{2}}^{k_{2}} \sim\left(k_{d}\right)^{d-1} .
$$

The operators $\hat{a}_{k}^{\dagger}, \hat{a}_{k}$, are the creation and annihilation operators for the eigenmodes corresponding to the eigennumber-sets $k$. They satisfy the usual algebra,

$$
\left[\hat{a}_{j}, \hat{a}_{k}^{\dagger}\right]=\delta_{j k}, \quad\left[\hat{a}_{j}, \hat{a}_{k}\right]=\left[\hat{a}_{j}^{\dagger}, \hat{a}_{k}^{\dagger}\right]=0 .
$$

Before continuing, we must note that the Hamiltonian (1) looks unbounded from below. However, this is not a problem. We must assume that the higher order stabilizing terms are included. As we shall show explicitly, such terms do not change the essence of the phenomenon. Therefore, we shall postpone the discussion of a Hamiltonian with the stabilizing terms included till later. For the time being, we shall ensure the boundedness of the energy by placing a cutoff on the maximal value of the angular momentum. Since the Hamiltonian (1) is particle number conserving, the angular momentum cutoff ensures boundedness of energy for any finite number of quanta. Later, we shall allow the particle number to be infinite, but in a special limit, which ensures that the energy is bounded from below.

Let us now rewrite the Hamiltonian in the language of the angular momentum modes. Integrating over the angular coordinates, we rewrite Hamiltonian in terms of the creation and annihilation operators,

$$
\hat{H}=\sum_{k} \epsilon_{k} \hat{a}_{k}^{\dagger} \hat{a}_{k}-g \Omega \sum_{s, k, q, r} C_{s k q r} \epsilon_{k} \epsilon_{r} \hat{a}_{s}^{\dagger} \hat{a}_{k}^{\dagger} \hat{a}_{q} \hat{a}_{r},
$$

where we have introduced a notation: $C_{s k q r} \equiv$ $\int d^{d} \Omega Y_{s}^{*} Y_{k}^{*} Y_{q} Y_{r}$. 
We shall now impose a cutoff that limits the maximal value of the angular momentum, $k_{d} \leq k_{*}$. Then, according to the definition (4), the corresponding eigenvalue of the Laplacian, is given by:

$$
\epsilon_{*} \equiv \frac{\hbar^{2}}{2 m R^{2}} k_{*}\left(k_{*}+d-1\right) .
$$

Notice, our main result is insensitive to the precise value of $k_{*}$, as long as it is much larger than one. Equivalently, the only natural assumption we are making is that the cutoff level $\epsilon_{*}$ is much higher than the elementary level $\frac{\hbar^{2}}{2 m R^{2}}$. Its precise value is unimportant for our analysis.

Next, let us focus our attention onto the states in which only the $k=0$ mode is macroscopically occupied. That is, the corresponding occupation number is very high, $\left\langle\hat{a}_{0}^{\dagger} \hat{a}_{0}\right\rangle=N_{0} \gg 1$. In the same time, we restrict the occupation numbers of $k \neq 0$ modes to be much smaller. On such states, we can use the Bogoliubov approximation [10] and replace the operators $\hat{a}_{0}^{\dagger}, \hat{a}_{0}$ by the $c$-numbers, $\hat{a}_{0}^{\dagger}=\mathrm{e}^{-i \alpha} \sqrt{N_{0}}, \hat{a}_{0}=\mathrm{e}^{i \alpha} \sqrt{N_{0}}$, where $\alpha$ is an unimportant phase.

In order to reveal the existence of a highly degenerate quantum critical point, we shall take the following useful limit. Notice, for a fixed value of $k_{*}$, we are left with two independent control parameters: The occupation number $N_{0}$ and the coupling strength $g$. Using these two control parameters, we shall take the following double-scaling limit:

$$
N_{0} \rightarrow \infty, \quad g \rightarrow 0, \quad g N_{0}=\text { finite }
$$

For the time being, we shall keep $\left(g N_{0}\right)$ as the remaining free control parameter.

In order to understand what happens with the interactions terms in the limit (9), let us split the sum into the pieces containing two, one or none of the zero-mode operators $\hat{a}_{0}^{\dagger}, \hat{a}_{0}$ respectively. Notice, none of the terms can contain more than two zero-mode operators, because they carry zero angular momentum. Thus, the second sum in (7) can be rewritten in the following form,

$$
\begin{aligned}
\text { Int terms }= & -g \hat{a}_{0}^{\dagger} \hat{a}_{0} \sum_{k \neq 0} \epsilon_{k}^{2} \hat{a}_{k}^{\dagger} \hat{a}_{k} \\
& -g \hat{a}_{0} \Omega \sum_{s, k, r \neq 0} C_{s k 0 r} \epsilon_{k} \epsilon_{r} \hat{a}_{s}^{\dagger} \hat{a}_{k}^{\dagger} \hat{a}_{r} \\
& -g \hat{a}_{0}^{\dagger} \Omega \sum_{k, q, r \neq 0} C_{0 k q r} \epsilon_{k} \epsilon_{r} \hat{a}_{k}^{\dagger} \hat{a}_{q} \hat{a}_{r} \\
& -g \Omega \sum_{s, k, q, r \neq 0} C_{s k q r} \epsilon_{k} \epsilon_{r} \hat{a}_{s}^{\dagger} \hat{a}_{k}^{\dagger} \hat{a}_{q} \hat{a}_{r},
\end{aligned}
$$

where in the fist term we have used the property $C_{0 k 0 r}=\frac{\delta_{k r}}{\Omega}$. The sum is taken up to $k_{*}$.
Now, in the double-scaling limit (9) the last three terms vanish. Indeed, the second and the third terms scale as $\frac{1}{\sqrt{N_{0}}}$, whereas the last term scales as $\frac{1}{N_{0}}$. Therefore, the only surviving term is the first one. Thus, in the double-scaling limit (9), the Hamiltonian (7) takes the following simple form:

$$
\hat{H}=\sum_{k \neq 0}^{k_{*}} \epsilon_{k}\left(1-g N_{0} \epsilon_{k}\right) \hat{a}_{k}^{\dagger} \hat{a}_{k} .
$$

We shall now use the remaining control parameter $\left(N_{0} g\right)$ and take the limit,

$$
g N_{0} \rightarrow \frac{1}{\epsilon_{*}}
$$

but in such a way that the quantity

$$
\delta_{*} \equiv \epsilon_{*}\left(1-g N_{0} \epsilon_{*}\right)
$$

approaches zero from the positive side. Obviously, $\delta_{*}$ represents the energy gap of the level- $k_{*}$ modes.

The resulting Hamiltonian is,

$$
\hat{H}=\sum_{k \neq 0}^{k_{*}} \epsilon_{k}\left(1-\frac{\epsilon_{k}}{\epsilon_{*}}\right) \hat{a}_{k}^{\dagger} \hat{a}_{k} .
$$

Thus, we arrive to a one-parameter family of the theories labeled by $\epsilon_{*}$.

Let us examine the resulting spectrum of the theory. First of all, all the modes with $k=k_{*}$ become exactly gapless. The number of such modes is equal to the degeneracy of the eigenvalue $k_{d}=k_{*}$. According to (5) this degeneracy is given by $\mathcal{N}_{k^{*}} \sim k_{*}^{d-1}$ and thus scales as the area of a $d-1$ dimensional sphere. So does the number of the gapless modes:

$$
N_{\text {modes }} \sim\left(\frac{R}{L_{*}}\right)^{d-1}
$$

where $L_{*} \equiv \frac{\hbar}{\sqrt{2 m \epsilon_{*}}}$. That is, if we fix the energy level $\epsilon_{*}$ of the critical angular excitation and vary the radius of the $d$-sphere, the number of gapless modes that can fit in it, scales as $R^{d-1}$. Note, the choice of the scale $L_{*}$ is somewhat analogous to the choice of the Planck length in gravity.

At the same time, the modes with $k_{d}<k_{*}$, have positive energy gaps. For example, the modes with $k_{d}=k_{*}-1$, have the energy gaps of order $\sim \frac{\hbar^{2}}{m R^{2}} k_{*}$, which is much higher than the unit energy gap. Thus, the modes $k<k_{*}$ do not contribute into the microstate entropy of the system.

Hence, the dominant contribution to the microstate entropy is coming from the gapless modes, with $k=k_{*}$. By "mildly" populating these modes, we can create an 
exponentially large number of microstates. For example, the number of basic microstates in which the occupation numbers of $k \neq 0$-modes take only two possible values, $\left\langle\hat{a}_{k}^{\dagger} \hat{a}_{k}\right\rangle=0$ or 1 , is $\mathcal{N}_{\text {states }}=2^{N_{\text {modes }} \text {. The entropy resulting }}$ from such microstates scales as,

$$
\text { Entropy }=\left(\frac{R}{L_{*}}\right)^{d-1} \text {. }
$$

By allowing the higher occupation numbers of the gapless modes, e.g., up to $\left\langle\hat{a}_{k}^{\dagger} \hat{a}_{k}\right\rangle<n$, we can increase the entropy by a $\log (n)$ factor, but the area scaling remains intact.

Before concluding this section, we wish to make couple of comments.

First, when taking the limit in which some of the modes become gapless, we have to make sure that the limit is smooth. That is, the interaction terms must remain suppressed and be subdominant under the small excitations of the gapless modes. In other words, we must avoid a strong coupling problem.

The fact that in our case the limit is smooth, should have already been clear from the fact that the double-scaling limit (9) allows to eliminate interaction terms, prior to taking the limit (12), which diminishes the gap $\delta_{*}$ of the $k_{*}$-modes, without restoring the interaction terms in (10).

In other words, since the gap $\delta_{*}$ and the interaction terms are controlled by different parameters, for an arbitrarily small value of $\delta_{*}$, we can make the interaction terms arbitrarily weak by taking $N_{0}$ large enough (equivalently, taking $g$ small enough).

Second, let us remark, that although according to the Hamiltonian (14) all the degenerate microstates have zero energies, this should not create a false impression that we manage to store an unlimited information in the system, without paying an overall energy price. We must remember that the macrostate described by the large occupation number $N_{0}$ of the zero angular-momentum quanta, costs an "inert" rest mass energy $E_{0}=N_{0} m c^{2}$, where $c$ is the speed of light. In the nonrelativistic limit this gives an overall infinite constant. However, this constant is invariant under a redistribution of the total particle number $N_{0}$ among the different angular-momentum-modes, and therefore, plays no role in the microstate count. So, the criticality of the level $\epsilon_{*}$ is not eliminating this universal energy price that any system (including a black hole) must pay for achieving a large microstate entropy.

Nevertheless, the criticality phenomenon allows us to gain enormously in the density of states, i.e., fitting an exponentially large number of states within an infinitesimal energy gap, thanks to a large number of the emergent gapless modes.

This situation is analogous to what happens in black holes. There too, a large microstate entropy of a black hole demands a huge overall energy price in form of the rest mass energy of the black hole. In other words, having many degenerate black hole states is only possible if each of them is very heavy.

\section{III. $S_{3} \mathrm{CASE}$}

Due to an obvious phenomenological importance of a three-dimensional space, we shall present an explicit counting for $d=3$. The angular coordinates $\vartheta^{a}$ on a 3 -sphere can be taken as $(\chi, \vartheta, \phi)$, where $0 \leq \chi \leq \pi, 0 \leq \vartheta \leq \pi$, $0 \leq \phi \leq 2 \pi$. The interval and the volume elements are given by $d s^{2}=d \chi^{2}+\sin ^{2} \chi\left(d \vartheta^{2}+\sin ^{2} \vartheta d \phi^{2}\right)$ and $d^{3} \Omega=\sin ^{2} \chi d \chi \sin \vartheta d \vartheta d \phi$, respectively. The spherical harmonics $Y_{k l m}(\chi, \vartheta, \phi)$ are labeled by the three integers, satisfying the condition: $|m| \leq l \leq k=0,1,2, \ldots \infty$. These functions are the eigenmodes of the Laplace operator on an unit $S_{3}$, with the eigenstate equation given by, $\Delta_{3} Y_{k l m}=$ $-k(k+2) Y_{k l m}$. As usual, they form a complete orthonormal set. Now, using the properties of $Y_{\mathrm{klm}}$-functions and repeating all the above computations for $d=3$, we arrive to the following form of the Hamiltonian in the double-scaling limit:

$$
\hat{H}=\sum_{|m| \leq l \leq k=1}^{k_{*}} \epsilon_{k}\left\{1-g N_{0} \epsilon_{k}\right\} \hat{a}_{k l m}^{\dagger} \hat{a}_{k l m},
$$

where $\epsilon_{k} \equiv \frac{\hbar^{2}}{2 m R^{2}} k(k+2)$.

Again, taking the limit

$$
\delta_{*} \equiv \epsilon_{*}\left\{1-g N_{0} \epsilon_{*}\right\} \rightarrow 0^{+},
$$

we get the spectrum of the gapless modes $\hat{a}_{k l m}^{\dagger}, \hat{a}_{k l m}$ with $k=k_{*}$. Their number is given by the corresponding degeneracy of the eigenvalue $k_{*}$ and is equal to,

$$
N_{\text {modes }}=k_{*}\left(k_{*}+2\right)=\left(\frac{R}{L_{*}}\right)^{2} .
$$

Thus, the microstate entropy scales as an area of a two-sphere:

$$
\text { Entropy }=\left(\frac{R}{L_{*}}\right)^{2} \text {. }
$$

Notice, the gapless modes $\hat{a}_{k_{*} l m}^{\dagger}, \hat{a}_{k_{*} l m}$ satisfy all the requirements for being called the holographic degrees of freedom. In particular, they saturate almost the entire information storage capacity of the system. The quantum messages can be encoded into the basic states with different occupation numbers of these modes, $|0,0, \ldots 0\rangle$, $|1,0, \ldots 0\rangle,|0,1, \ldots 0\rangle, \ldots .,|1,1, \ldots 1\rangle, \ldots$, as well as in their superpositions. The superpositions formed by these states can be highly entangled and have a very high complexity. 


\section{BOUNDED HAMILTONIAN}

In order to avoid creating a wrong impression as if the emergence of the area law relies on a truncation of the angular-momentum tower, we shall consider an example of the bounded-from-below Hamiltonian, for which no angular-momentum cutoff is required for the stability.

We choose the Hamiltonian in the following form:

$$
\begin{aligned}
\hat{H}= & \int d^{d} \Omega\left\{-\hat{\psi}^{\dagger} \Delta \hat{\psi}-g \Omega\left(\hat{\psi}^{\dagger} \Delta \hat{\psi}^{\dagger}\right)(\hat{\psi} \Delta \hat{\psi})\right. \\
& \left.+\tilde{g} \Omega\left(\hat{\psi}^{\dagger} \Delta^{2} \hat{\psi}^{\dagger}\right)\left(\hat{\psi} \Delta^{2} \hat{\psi}\right)\right\}
\end{aligned}
$$

where $\tilde{g}$ is a new positive constant, of dimensionality of inverse energy-cubed. Again, this Hamiltonian possesses a quantum critical point at which a large number of gapless modes emerge, resulting in the area-law microstate entropy.

In order to see this, we again integrate over the space coordinates and take the double-scaling limit

$$
\begin{gathered}
N_{0} \rightarrow \infty, \quad g \rightarrow 0, \quad \tilde{g} \rightarrow 0 \\
g N_{0}=\text { finite }, \quad \tilde{g} N_{0}=\text { finite } .
\end{gathered}
$$

The resulting Hamiltonian has the following form:

$$
\hat{H}=\sum_{k \neq 0}^{\infty} \epsilon_{k}\left\{1-g N_{0} \epsilon_{k}+\tilde{g} N_{0} \epsilon_{k}^{3}\right\} \hat{a}_{k}^{\dagger} \hat{a}_{k} .
$$

The difference from the previous case is that here we are left with two control parameters: $g N_{0}$ and $\tilde{g} N_{0}$. From the above expression it is clear that the boundedness of the Hamiltonian no longer requires a cutoff, since the modes with $\epsilon_{k} \rightarrow \infty$ are stable.

We shall now impose the constraint on the Hamiltonian (23) to be semipositive definite. Then, the quantum criticality is reached whenever the Hamiltonian touches zero for some $\epsilon_{k}=\epsilon_{*}$. Such a situation is achieved when the two control parameters, $\left(g N_{0}\right)$ and $\left(\tilde{g} N_{0}\right)$, take the following values:

$$
\left(g N_{0}\right)=\frac{3}{2} \frac{1}{\epsilon_{*}}, \quad\left(\tilde{g} N_{0}\right)=\frac{1}{2} \frac{1}{\epsilon_{*}^{3}} .
$$

With the above choice, the eigenvalues of the Hamiltonian (23) are semipositive definite and touch zero for $\epsilon_{k}=\epsilon_{*}$. This can be easily visualized by using (24) and rewriting the Hamiltonian (23) in the form

$$
\hat{H}=\sum_{k \neq 0}^{\infty} \frac{\epsilon_{k}}{2}\left(\frac{\epsilon_{k}}{\epsilon_{*}}-1\right)^{2}\left(\frac{\epsilon_{k}}{\epsilon_{*}}+2\right) \hat{a}_{k}^{\dagger} \hat{a}_{k} .
$$

In this case, only the modes with $\epsilon_{k}=\epsilon_{*}$, become gapless, while all the other modes $\epsilon_{k} \neq \epsilon_{*}$ have the positive energy gaps and are stable.
Thus, once again, the choice of the control parameters given by (24), defines a one parameter family of theories parameterized by the scale of criticality $\epsilon_{*}$. Notice, the critical level $\epsilon_{*}$ is no longer a cutoff of the tower, but an arbitrary marker that separates the modes into the highlevel (microscopic) and the low-level (macroscopic) ones. Its value can be chosen arbitrarily. In this sense, the corresponding length-scale $L_{*} \equiv \frac{\hbar}{\sqrt{2 m \epsilon_{*}}}$, defined earlier, plays the role of a nongravitational "Planck length" for this toy nonrelativistic model. ${ }^{1}$

Of course, it is natural to assume that $\epsilon_{*}$ is much higher than the unit gap $\frac{\hbar^{2}}{2 m R^{2}}$. Then, repeating the previous counting, we immediately obtain that the number of the gapless modes and the entropy of the resulting microstates are given by the Eqs. (15) and (16), respectively. Both of these quantities scale as the area of a $d$-1-dimensional sphere of radius $R$.

\section{SUMMARY OF THE MECHANISM}

We are now in the position to summarize the essence of the considered mechanism. It consists of two crucial ingredients. These are: (1) The large- $N_{0}$ criticality; and (2) the level-degeneracy due to the spherical symmetry.

Let us elaborate on the above points.

First of all, the high occupation numbers of some of the low-level modes-due to their attractive couplings to the high-level ones-diminish the gaps of the latter modes. Indeed, as we saw, for a high occupation number $N_{0}$ of the 0 -level mode and for a certain value of the criticality parameter, the effective energy gaps of $k_{*}$-level modes were lowered to zero. Notice, in the state $N_{0}=0$, the energy gaps for the same $k_{*}$-modes would be enormous, $=\epsilon_{*}$. However, in the state in which $(k=0)$-level mode is critically occupied, the gaps of $k_{*}$-modes collapse to zero.

In a nutshell, a critical occupation of the low-level modes collapses the gaps of the high-level ones.

Once this is appreciated, the entropy counting follows solely from the degeneracy of the level $\epsilon_{*}$, which is fully controlled by the angular momentum conservation and the spherical symmetry of the problem.

In is natural to hypothesize that the above phenomenon is indeed taking place in black holes, provided we assume that the black holes are the states in which some of the soft gravitons are macroscopically occupied to a critical level $[7,11]$. Then, the high occupation number of the soft gravitons, suppresses the energy gaps of the Planck wavelength ones, resulting into the area law entropy.

Notice: This is a trade-off. By putting a lot of energy in high occupation number of soft gravitons, the black hole makes the excitations of Planck-wavelength gravitons very cheap.

\footnotetext{
${ }^{1}$ Of course, the true gravitational Planck length vanishes in nonrelativistic limit.
} 


\section{DISCUSSION}

We have shown that a simple nonrelativistic quantum system living on a $d$-dimensional sphere and describing a bosonic field with an attractive self-coupling, possesses a quantum critical state with an exponentially enhanced degeneracy of microstates. Remarkably, the entropy of these microstates is given by an area of a $d$-1-dimensional sphere.

We must note, that thanks to double-scaling limit, the entropy-counting is exact and is not corrected by quantum fluctuations. Of course, once we depart from this limit, we expect the finite gaps to develop. However, they will be suppressed by $\frac{1}{N_{0}}$ and thus will be under control in the large- $N_{0}$ regime.

This model also gives a precise meaning to the concept of holography. Indeed, the gapless modes that emerge at the critical point, by all measures, represent the holographic degrees of freedom, with their number scaling as area of a sphere, of one dimension lower than the bulk space.

How can we interpret the above result? There are at least three different points of view that one could take.

The first view is that the above quantum system does capture some fundamental properties of quantum gravity, and therefore, the area law of entropy is of gravitational type. In particular, this point of view is supported by the fact that the self-interaction of the bosonic field is both attractive and momentum-dependent, something that is highly reminiscent of gravity.
The second view is that the phenomenon of the enhancement of entropy and its scaling according to the area law goes well beyond gravity, and we are observing one such example.

These two viewpoints are not interexclusive and they are the two components of the hypothesis of [7]. This is also our point of view. That is, we think that the area law scaling of Bekenstein entropy originates from quantum critical state reached due to the high occupation number of attractive gravitons. However, at the same time, there can exist other systems of nature that can exhibit a similar behavior.

Finally, there is a third view stating that the aboveobserved area-law-type entropy is just a remarkable coincidence, unrelated to the black hole physics. This is a fully legitimate point of view, although not our favorite one. However, if it were true, it could teach us an equally important lesson. This lesson would be that black holes may not possess a complete monopoly on holographic scaling of entropy.

\section{ACKNOWLEDGMENTS}

It is a pleasure to thank Lasha Berezhiani, Cesar Gomez and Sebastian Zell for discussions. This work was supported in part by the Humboldt Foundation under Humboldt Professorship Award, ERC Advanced Grant No. 339169 "Selfcompletion", by TR 33 "The Dark Universe", and by the DFG cluster of excellence "Origin and Structure of the Universe."
[1] J. D. Bekenstein, Black holes and entropy, Phys. Rev. D 7, 2333 (1973).

[2] G.'t Hooft, Dimensional reduction in quantum gravity, arXiv:gr-qc/9310026; L. Susskind, The World As A Hologram, J. Math. Phys. (N.Y.) 36, 6377 (1995).

[3] J. M. Maldacena, The large N limit of superconformal field theories and supergravity, Adv. Theor. Math. Phys. 2, 231 (1998).

[4] S. S. Gubser, I. R. Klebanov, and A. M. Polyakov, Gauge theory correlators from non-critical string theory, Phys. Lett. B 428, 105 (1998).

[5] E. Witten, Anti-de Sitter space and holography, Adv. Theor. Math. Phys. 2, 253 (1998).
[6] L. Susskind and E. Witten, The holographic bound in antide Sitter space, arXiv:hep-th/9805114.

[7] G. Dvali and C. Gomez, Black holes as critical point of quantum phase transition, Eur. Phys. J. C 74, 2752 (2014).

[8] A. Strominger and C. Vafa, Microscopic origin of the Bekenstein-Hawking entropy, Phys. Lett. B 379, 99 (1996).

[9] G. Dvali, A. Franca, C. Gomez, and N. Wintergerst, NambuGoldstone effective theory of information at quantum criticality, Phys. Rev. D 92, 125002 (2015).

[10] N. Bogoliubov, On the theory of superfluidity, J. Phys. 11, 23 (1947).

[11] G. Dvali and C. Gomez, Black Hole's Quantum N-Portrait, Fortschr. Phys. 61, 742 (2013). 